\title{
TECNOLOGIA DE INFORMAÇÃO E SUA INFLUÊNCIA SOBRE OS RUMOS DA COMERCIALIZAÇÃO DE PRODUTOS (1)
}

\author{
Lilian Cristina Anefalos \\ J osé Vicente Caixeta Filho
}

\begin{abstract}
Resumo
Mostra a importância da tecnologia de informação na atualidade, com ênfase em alguns setores chaves ligados à comercialização de produtos. Apresenta os principais avanços e limitações da combinação de recursos de telecomunicações e de tecnologia de informação nos principais elos de algumas cadeias de comercialização. Apesar do grande potencial dessa nova tecnologia é importante que haja cooperação entre os diversos setores dessa cadeia para permitir sua plena atuação em todos os ramos da comercialização.
\end{abstract}

\section{Palavras-chave}

Satélite; Tecnologia de Informação;Acesso à Informação; Comercialização; Transporte.

\section{INTRODUÇÃO}

\footnotetext{
${ }^{1}$ Este artigo faz parte do projeto de dissertação de mestrado em Economia Aplicada da primeira autora na ESALQ/USP, sob orientação do segundo autor.
}

O acesso à informação tem se constituído em um poderoso instrumento para as empresas tanto do setor público como do privado, auxiliando-as na tomada de decisão. Com isto, cada vez mais têm sido feitos investimentos em tecnologias de ponta, a fim de garantirem a sobrevivência no mercado e, principalmente, gerarem serviços e produtos de qualidade e ao menor custo, através da redução dos prazos de entrega e minimização de falhas em todo o processo produtivo e de distribuição.

Nesse sentido, muitas contribuições científicas têm procurado explicar melhor o papel das tecnologias de informação. Porém, apesar de envolver, geralmente, grandes volumes de capital para sua implantação e manutenção, houve poucos avanços concretos no dimensionamento econômico e financeiro do seu exato valor dentro das empresas, ou seja, relacionado às oportunidades de investimento das empresas, ou mesmo sobre a eficiência desses novos sistemas sobre os seus usuários.

Apesar disso, diversos setores da economia já estão se dedicando à introdução de novos sistemas ou mesmo de um parque tecnológico (ou seja, de uma gama muito cariada de novas tecnologias, interligadas através de vários sistemas), visando o aumento da competitividade frente aos concorrentes, procurando se diferenciar junto aos 
consumidores ou usuários.

Dessa forma, o objetivo deste trabaIho é o de apresentar um panorama geral da tecnologia de informação, focando principalmente alguns setores chaves relacionados à comercialização, a fim de mostrar seu desenvolvimento e suas limitações frente aos novos avanços via satélite.

O trabalho está dividido em quatro partes incluindo esta introdução. Na segunda parte a tecnologia de informação será descrita e conceituada, assim como abordados os seus principais avanços; a terceira parte trará algumas diretrizes das aplicações espaciais da tecnologia de informação para os setores que compõem a cadeia de comercialização; na quarta parte serão apresentadas considerações finais.

\section{ASPECTOS GERAIS DA TECNOLOGIA DE INFORMAÇÃO} (TI)

A tecnologia da informação (TI) se originou nas indústrias de informática e de telecomunicações, ligada à criação, transmissão, acumulação e processamento de dados. Para fins de competitividade ${ }^{2}$, há três tendências que devem ser consideradas: digitalização da rede de telecomunicações, através de centrais digitalizadas, de fibra ótica e de satélites; telecomunicações sem fio, através de telefonia celular, de satélites de baixa órbita e de terminais de comutação; inovações em terminais e computadores conectados às redes (Tigre, Rovere e Fagundes, 1995).

De acordo com Silva (1995), a tecnologia de informação permite também automatizar processos agrícolas para viabilizar o controle, o manejo e a comercialização de produtos, sem prejuízo de escala, e permitindo um atendimento personalizado aos clientes.

Assim, o conceito de TI pode ser aplicado de diversas maneiras. Laurindo (1995) utilizou a definição de $\mathrm{TI}$ adaptada às empresas ${ }^{3}$, restringindo-a a três áreas: informática, sistemas de informação, desenvolvimento de sistemas.

Outra forma, citada por Bowersox \& Closs (1996), seria relacionar as seguintes aplicações no campo da logística ${ }^{4}$ : troca de dados eletrônicos (EDI); computadores pessoais; inteligência artificial (sistemas especialistas, tradutores de linguagem natural, redes neurais, robótica, reconhecimento de discurso, visão 3D); comunicações (freqüência de rádio, satélite, processamento de imagens e imagens eletrônicas); código de barras e digitalização de imagens (scanning).

Deve-se, entretanto, enfatizar a importância da área de comunicação, ou mais especificamente, as inúmeras aplicações advindas dos satélites, que têm causado profundas transformações estruturais em nível mundial.

Para acompanhar o processo de mudança de comportamento do mercado, tem havido uma grande tendência de reestruturação da filosofia das empresas em todo o mundo, inclusive no Brasil, com a finalidade de garantirem seu espaço. Segundo Genezini (1994)

\footnotetext{
${ }^{2}$ Competitividade, segundo Long (1996), se refere à sobrevivência das firmas no longo prazo através de custos baixos e de lucros compatíveis. Ela se diferencia do conceito de competição perfeita e, apesar de ser altamente subjetiva, a competitividade pode ser medida através da elasticidade e dos lucros normais.

${ }^{3}$ Segundo Weil (1992), citado por Laurindo (1995), TI se refere ao uso e à inovação de sistemas de informação, de hardware, de sistemas de automa-
} 
TABELA 1 - Evolução do Papel da Tecnologia de Informação (anos 70 a 90)

\begin{tabular}{|l|l|l|}
\hline Anos 70 & Anos 80 & Anos 90 \\
\hline Introdução & Integração & Infra-estrutura \\
\hline Processamento de dados & Sistemas de informação & $\begin{array}{l}\text { Tecnologia de } \\
\text { informação }\end{array}$ \\
\hline $\begin{array}{l}\text { "Para suportar o } \\
\text { negócio..." }\end{array}$ & $\begin{array}{l}\text { "Para suportar o } \\
\text { negócio..." }\end{array}$ & $\begin{array}{l}\text { "Para transformar o } \\
\text { negócio..." }\end{array}$ \\
\hline
\end{tabular}

Fonte: Andersen Consulting, transcrito por morgado (1995, p.16).

do (1995), o papel da TI tem acompanhado essa evolução, influenciando cada vez mais a organização das estratégias de negócios (Tabela 1 , no alto da página).

Paralelamente, o estudo desenvolvido por Laurindo (1995) mostrou que a eficácia da TI, com relação a fatores organizacionais, necessita da participação de profissionais da área de informática e também dos usuários. Comparando a TI com os sistemas tradicionais, de acordo com Walton (1993), pode-se dizer que essas novas tecnologias exigem uma interação maior entre os agentes, principalmente em função de três fatores: distribuição de tarefas, aprendizagem e motivação

ção, considerando também serviços e usuários e as relações complexas entre todos esses componentes. A referência completa da citação é a seguinte: Weil, P. The relationship between investment in information technology and firm performance: a study of the valve manufacturing sector. Information Systems Research, v.3, n.4, p.307-333, Dec. 1992. ${ }^{4}$ Logística é definida, segundo o Council of Logistics Managementem 1991 (citado por Bowersox \& Closs, 1996, p.4), como "o processo de planejamento, implementação e controle eficiente do fluxo e armazenamento de mercadorias, serviços e informação transmitida do ponto de origem para o consumo, de acordo com as exigências do consumidor." interna, cujo bom andamento é imprescindível para que a reestruturação das empresas seja bem sucedida.

Em relação aos usuários ou clientes, Lambert (1995) ressalta a sua importância dentro dessa nova dinâmica tecnológica, e recomenda para que se efetuem programas de pesquisa quanto às suas preferências ou necessidades, e só a partir de então se instituam programas de serviço ao cliente.

Há um aspecto interessante, estudado pelo Instituto de Pós-Graduação e Pesquisa da Universidade Federal do Rio de Janeiro e publicado no artigo $O$ Cliente (1996), que ressalta ser o cliente a variável de decisão de compra que mais tem crescido em relação às outras mais tradicionalmente citadas: produto, preço e promoção e propaganda. O serviço ao cliente obteve uma variação positiva de $24,3 \%$ em sua participação na compra, comparando-se 1994 a 1995, bastante significativa em relação às demais variáveis (Tabela 2). Os clientes demonstraram uma exigência crescente nos serviços, não sendo, entretanto, correspondida pelos fornecedores no período analisado, que obtiveram um percentual

\footnotetext{
${ }^{5}$ Genezini, S. Reengenharia - visão global. Palestra da Andersen Consulting na FEBRABAN. Maio. 1994. (Anotações da palestra).
} 


\section{TABELA 2 - Participação Relativa de Atributos na Decisão de Compra pelo Cliente (1994-1995)}

\begin{tabular}{|l|c|c|r|}
\hline Variáveis & \multicolumn{2}{|c|}{ Participação relativa (\%) } & Variação (\%) \\
\hline & $\mathbf{1 9 9 4}$ & $\mathbf{1 9 9 5}$ & 2,9 \\
\hline Produto & 30,7 & 31,6 & $-6,8$ \\
\hline Preço & 38,4 & 35,8 & $\mathbf{2 4 , 3}$ \\
\hline Serviço ao cliente & $\mathbf{1 4 , 4}$ & $\mathbf{1 7 , 9}$ & $-10,4$ \\
\hline Promoção e Propaganda & 16,4 & 14,7 & \\
\hline Total & 100,0 & 100,0 & \\
\hline
\end{tabular}

Fonte: O Cliente (1996, p.55)

crescente de 1994 a 1995 de insatisfação do cliente pelos seus serviços prestados ${ }^{6}$.

\subsection{Principais Avanços da TI}

Segundo Weitzen (1991) e Alvin Tofler em palestra na Comdex, 1993, citado por Oxner \& Charlab (1995), o mundo já passou por duas eras: a primeira, agrária, baseada no potencial humano; a segunda, industrial, centrada em energias mais baratas.

Atualmente, está sendo formada uma nova era, da informação, tida como um fator crítico e decisivo no desenvolvimento econômico e tecnológico das nações ${ }^{7}$, baseada numa maior velocidade no fluxo de informações, através do emprego de novas tecnologias, onde a sociedade está centrada na economia da informação ${ }^{8}$.

Conforme Strassmann (1985), na era eletrônica as organizações eficazes têm se sobressaído mais devido ao seu maior envolvimento com cooperações técnica e política e com a comunicação.

Nessa revolução da informação, se- gundo Weitzen (1991), um dos princípios básicos de sucesso é "fazer mais, por menos", ou seja, fornecer um melhor serviço com menos recursos. Para isso, há estratégias para administrar as informações como: utilizá-las de banco de dados, personalizá-las, facilitar o seu acesso, agilizar o seu fluxo, reorganizá-las, fornecer sua entrega 24 horas por dia e integrar o seu triângulo ${ }^{9}$.

Fernandes \& Alves (1992) enfatizam que há alguns fatores críticos que afetam o sucesso da TI nas empresas. Entre eles, podem ser relacionados: inércia organizacional, ou seja, inovações não ime-

\footnotetext{
${ }^{6}$ Essa pesquisa foi realizada em dois períodos: início do Plano Real, em 1994, e no final de 1995, e avaliou a relação entre a indústria de bens de consumo, o atacado e o varejo. O serviço ao cliente foi medido através de nove dimensões, sendo que destas, três tiveram sua importância elevada em relação às demais nos dois anos analisados. A saber: disponibilidade de produto, consistência de prazo de entrega e tempo de ciclo de pedido, com variação da insatisfação de $48,4 \%, 90 \%$ e $136,7 \%$, respectivamente (O Cliente, 1996).

${ }^{7}$ De acordo com Mesquita (1995), em 1972 a sociedade da informação gerava US $\$ 40$ bilhões; dados de 1992 estimam uma economia de US\$ 140 biIhões nesse setor.
} 
diatas com relação ao aumento da competitividade; confiança exagerada dos dirigentes da empresa em que a TI resolverá todos os problemas; alta rigidez na estrutura organizacional, impedindo a introdução de novas tecnologias; apoio pequeno da alta gerência da empresa; não avaliação dos impactos das alterações ocorridas na empresa após a implantação da TI; treinamento descontínuo dos funcionários.

Como conseqüências diretas desse quadro, segundo os mesmos autores, ocorrerão: atraso tecnológico em relação aos concorrentes, colocando produtos menos competitivos no mercado; altos custos na implementação da TI, podendo inviabilizar a sua continuidade na empresa; equipe não sintonizada para o trabalho conjunto.

Em relação à área de marketing, segundo Gonçalves \& Gonçalves Filho (1995), a TI é vista como um elemento essencial para que as firmas obtenham vantagem competitiva $^{10}$ sobre as demais, reduzindo seus custos e diferenciando seus produtos no mercado.

Nesse sentido, os mesmos autores relacionam quatro grupos de variáveis que direcionam os benefícios da TI: praça, onde as compras de mercadorias são realizadas com mais intensidade, apenas entre empresas que se utilizam da EDI, e a sua distribuição é feita com acompanhamento on-

\footnotetext{
${ }^{8}$ Segundo Malin (1994), economia da informação conceitua a informação como um recurso, reagrupando a estrutura produtiva da sociedade, de acordo com novos critérios de classificação, em quatro setores: produção, processamento, distribuição de produtos e serviços de informação.

${ }^{9}$ No triângulo da informação, segundo Weitzen (1991), interagem três elementos: consolidar os recursos para melhorar as informações, identificar maneiras para que elas sejam pagas imediatamente e potencializar as informações, os produtos e os serviços para que sejam distribuídos em todo o mundo.
}

line, de todo o trajeto dos produtos até o destino final; promoção/vendas, aperfeiçoada através de notebooks, expert systems ${ }^{11}$, sistemas de vídeo por computador, código de barra, cartões magnéticos, smart cards ${ }^{12}$; produto, com serviços de atendimento ao cliente, controle de qualidade; produtividades operacional (sistemas de otimização, reengenharia, qualidade), gerencial (sistemas de informação para executivos - EIS, especializados e de treinamento), de escritório (PCs, automação de serviços, correio eletrônico) e de informática (linguagens mais poderosas, terceirização), relacionadas ao aumento da produtividade e à redução dos custos.

Outro ponto positivo que também deve ser considerado é que a TI tem permitido, em organizações bem adaptadas a novas tecnologias, trabalho conjunto de equipes em espaços físicos distantes, interligados pelas chamadas redes virtuais, viabilizando a continuidade das atividades das empresas (CUNHA OLIVEIRA, 1996).

Além disso, McGaughey Jr., Snyder e Carr (1994) enfatizam que os riscos também devem ser analisados pelas empresas com relação à introdução de inovações tecnológicas. Desta forma, a sua avaliação deveria ser conduzida na etapa de planejamento da TI.

\footnotetext{
10 Vantagem competitiva, segundo McGaughey Jr., Snyder e Carr (1994, p.273), diz respeito à "habilidade de se sobressair no mercado devido a: preço, produto, nível de serviço ou desempenho".

${ }^{11}$ Expert systems agem como se fossem especialistas em determinados assuntos, auxiliando o seu usuário no processo de tomada de decisão (GONÇALVES, GONÇALVES FILHO, 1995).

${ }^{12}$ Smart cards são cartões magnéticos inteligentes que armazenam entre outras informações, dinheiro, que pode ser transferido instantaneamente (GONÇALVES, GONÇALVES FILHO, 1995).
} 


\section{DIRETRIZES DA TI EM ALGUNS SETORES IMPORTANTES PARA A COMERCIALIZAÇÃO}

A comercialização se refere ao "conjunto de atividades realizadas por instituições que estão empenhadas na transferência de bens e serviços desde o ponto de produção inicial até que eles atinjam o consumidor final..." (PIZA \& WELSH ${ }^{13}, 1968$, p.1, citados por BARROS, 1987).

Dentro desse processo, há algumas funções importantes desempenhadas por cada um dos agentes, desde produtores rurais (ou suas coordenações/integrações, como por exemplo, cooperativas, agroindústrias), atacadistas até varejistas, envolvidos em etapas intermediárias como armazenamento, transporte e processamento, para atingir o último elo da cadeia, ou seja, os consumidores (BARROS, 1987).

De acordo com Brito (1996, p.18), “a tecnologia de informação pode ser uma alavanca do processo de integração da cadeia de suprimento. Ela não apenas substitui os tipos anteriores de métodos de comunicação, mas leva a alterações na rotina de trabalho das pessoas".

Davenport (1994) destaca, dentre as diversas aplicações da TI, as relacionadas ao:

- gerenciamento de pedidos, a fim de agilizar a entrega, aumentando a satisfação do usuário e reduzindo custos. Neste caso, deve-se ressaltar as comunicações de voz e a troca eletrônica de dados (EDI);

\footnotetext{
${ }^{13}$ Piza, C. T.; Welsh, R. W. Introdução à análise da comercialização. 1968. (Série Apostila, 10).
}

- processo logístico, ou seja, a administração de pedidos, estoques, materiais e serviço de entrega através de: sistemas de localização, para que haja um melhor planejamento do itinerário dos caminhões, navios; sistemas de gerenciamento de bens, através de depósitos inteligentes, monitorando o estoque de mercadorias nas instalações da empresa; sistemas de planejamento logístico, através de sistemas especialistas, reengenharia de processos, telemetria, usada em tecnologias sem fio para fazer o acompanhamento de processos à distância.

Uma das áreas da TI que mais tem se expandido atualmente tem sido a telemática, ou seja, área que combina recursos de telecomunicações e da TI (Slats et al, 1995) e, portanto, as suas características e o seu potencial de aplicação para a comercialização serão detalhadamente expostos. Deve-se ressaltar que a Embratel é a empresa que intermedia a utilização dos satélites pelas empresas no Brasil.

A Internet é uma das alternativas mais acessíveis a todos os elementos da cadeia, e possui vários tipos de serviços que podem ser utilizados diariamente, incluindo os que obtêm informações mais específicas, como por exemplo, boletins meteorológicos (inclusive direcionados para a agricultura ${ }^{14}$ ), cotações de preços e índices, recomendações de rotas menos congestionadas, compra de produtos/serviços, entre outros.

A EDI (Eletronic Data Interchange / Troca Eletrônica de Dados) é uma outra tecnologia que possui um potencial muito grande nas relações entre as empresas, quanto à troca das mais diversas informações, através de voz, dados, ou vídeo. É administrada por VANs (Value Added Networks), que oferecem serviços padronizados mundialmente, podendo-se citar: 
cotação eletrônica de preços, troca eletrônica de documentos, transações financeiras, reposição automática de estoques, procurando atingir uma completa integração logística (VANS, 1996).

Segundo Streeter, Sonka e Hudson (1991), há uma crescente conscientização das grandes empresas atacadistas e varejistas, inclusive das relacionadas ao agribusiness, em melhorar suas estratégias de marketing junto aos consumidores, facilitando os fluxos de informação e coordenação da produção, através do emprego de TI.

Segundo Graham \& Nazem (1996), com o emprego da EDI junto aos fornecedores, na indústria de alimentos há perspectivas de redução de capital de giro, diminuição dos estoques e utilização mais eficiente de espaço, que conduzirão a produtos mais frescos, menores custos e aumento nas vendas.

Entre as pequenas e médias empresas poucos estudos foram feitos para avaliar os fatores de sucesso para obter vantagens com o emprego da EDI. Taymond \& Bergeron (1996), utilizando uma amostra de 39 firmas canadenses, obtiveram que apenas uma parcela bem pequena tem conseguido um lucro real com a EDI, e que muitas delas ainda consideram esta tecnologia como um custo adicional para concretizar os negócios.

Essa nova tecnologia é ainda muito

\footnotetext{
${ }^{14} \mathrm{Na}$ agricultura brasileira merece destaque o desenvolvimento de sistemas de informação geográfica (SIG), que manipulam dados espaciais de diversas fontes como mapas, imagens, cadastros, e que têm permitido se conhecer melhor todo o território nacional, e se estudar melhor os recursos ambientais e aspectos meteorológicos. Entre as instituições brasileiras especializadas nesse tipo de atividade de pesquisa, pode-se citar o INPE - Instituto de Pesquisas Espaciais (Alves, 1990).
}

incipiente no Brasil, destacando-se, no uso da EDI, o setor bancário e algumas grandes indústrias, como a automobilística e as caracterizadas como principais fornecedores, entre elas: Brahma, Refinações de MiIho Brasil e Makro (BLANCO, 1996).

Mais recentemente tem havido um estreitamento muito forte na relação entre indústria e pequenos varejistas, em nível nacional, viabilizando novas fronteiras de comercialização, até então, não devidamente exploradas pela inexistência de um fator decisivo de interação entre eles ${ }^{15}$.

Paralelamente à EDI, segundo Blanco, há uma aplicação logística mais recente no varejo, o quick-response, que permite se estabelecer uma comunicação on-line entre a equipe de vendas e os fornecedores, sejam eles indústrias ou atacadistas.

Além disso, dentre os setores chaves para a comercialização em que há grandes preocupações tecnológicas está o de transporte. Apesar de seus investimentos terem se reduzido, segundo a Confederação $\mathrm{Na}$ cional da Indústria (1995), em mais de 10\% (dados de 1993, em relação à 1980), principalmente em função da diminuição de projetos do setor público, tendem a vislumbrar um grande potencial nos próximos anos devido às novas perspectivas de aplicações logísticas modernas, de automação e de segurança.

Caixeta Filho (1996) ressalta que a participação ativa do transporte dentro das estruturas organizacionais de outros setores, faz com que ele se torne imprescindível para as demais atividades, não poden-

\footnotetext{
15 Neste caso, pode-se citar um projeto recente, o Programa Sincovaga, unindo o Sindicato do Comércio Varejista de Gêneros Alimentícios (Sincovaga) e a Fundação Instituto de Administração da Universidade de São Paulo (FIA/USP) para viabilizar o
} 
do mais ser tratado isoladamente.

O transporte rodoviário, que representa $70 \%$ do total dos modais usados no Brasil (ESTRADAS, 1996), é o que expressa grandes expectativas quanto a essas transformações.

Mais especificamente em relação às transportadoras, há expectativas de que a forte interação, segundo Feliciano Neto (1996), entre logística, automação e TI no setor de transportes, conduza a uma minimização de custos, e permita que as organizações adquiram vantagens competitivas.

Como reflexo direto disso, a tendência é a redução do número de transportadoras, sobressaindo-se apenas as de maior porte. Estas é que irão interagir em nível nacional, com os embarcadores e com as transportadoras menores, e em nível regional, através de acordos operacionais (UM ANO, 1997).

Nesse sentido, os sistemas de rastreamento $^{16}$, via satélite, têm se destacado como uma das tecnologias de ponta do transporte rodoviário, que além de propiciar a comunicação móvel, permitem também a localização de objetos em movimento (PIQUINI, 1990). Nos Estados unidos são utilizados por mais de $50 \%$ das empresas transportadoras, segundo dados da Telecom Publishing Group (TECNOLOGIA, 1995); o seu preço para o Brasil é, ainda, elevado ${ }^{17}$. No entanto, segundo Lopes (1996), o aumento da segurança, e sua interface com os roteirizadores ${ }^{18}$, têm se constituído em grandes incentivos para as

comércio entre o pequeno varejo e a indústria através do sistema de compras via EDI. Esta estratégia pretende resolver problemas de distribuição de produtos, prazos de entrega, tornando os preços de mercado mais competitivos (Varejistas, 1996). empresas nacionais introduzirem essa tecnologia.

\section{CONSIDERAÇÕES FINAIS}

É importante ressaltar que a tecnologia de informação engloba um conjunto de ferramentas que, entre os seus maiores benefícios, servirão para melhor conduzir todas as etapas da comercialização, procurando substituir com mais eficiência mecanismos operacionais não muito eficazes. Um dos meios que serão gradativamente mais explorados serão os satélites, que em pouco tempo já obtiveram avanços significativos em inúmeros setores da economia, nos quais é importante destacar a EDI e o sistema de rastreamento, com um potencial de utilização cada vez maior, principalmente devido à abertura dos mercados e à intensificação do processo de globalização em todos os países.

No entanto, nem todos os problemas podem ser solucionados pela $\mathrm{Tl}$, como é o

\footnotetext{
${ }^{16}$ Esses sistemas, segundo Lopes (1996), coletam periodicamente as posições dos veículos através do GPS (Global Position System), por satélite, e transferem esses dados para uma estação interna, que os retransmite para uma estação intermediária (opcional) e finalmente para o usuário. A comunicação do usuário com o veículo é efetuada pela seqüência inversa.

17 Entre as cinco empresas fornecedoras desse sistema no Brasil, o preço do software de comunicação varia entre $R \$ 6$ mil e $R \$ 8$ mil por veículo. Há, porém, outras despesas para os usuários que não foram consideradas nesse preço, como tarifas mensais e custos de instalação do equipamento (Lopes, 1996).

18 Segundo Oliveira (1996), a corretora de seguros Pamcary, especializada na prevenção e no combate ao roubo de cargas, controla duzentas mil viagens de caminhões de transporte, em média, por mês, com este sistema.
} 
caso do transporte físico de produtos, que depende em grande parte de melhorias das vias para escoamento da produção para as diversas regiões do país ou do exterior.

A deterioração das estradas, no caso do transporte rodoviário, por exemplo, é um fator decisivo para que haja um aumento nos custos de transferência, devido aos atrasos na entrega e aos roubos, com prejuízos ou perdas nas cargas transportadas (principalmente em relação a produtos perecíveis).

O estado geral das rodovias se torna cada vez mais crítico diante da tendência do comércio, principalmente no varejo, em reduzir cada vez mais seus estoques e necessitar da pronta entrega das mercadorias em falta para atender às necessidades dos consumidores (MAIS, 1996).

Além disso, para que haja um melhor aproveitamento de todas as vantagens das novas tecnologias, é imprescindível que as empresas se reestruturem internamente, e se capacitem adequadamente tanto em equipamentos como em recursos humanos, para que enfrentem mais efetivamente os concorrentes, e possam se diferenciar das demais empresas do mercado. Um papel muito importante para a programação e distribuição das atividades será o da logística.

Nesse sentido, é preocupante o desenvolvimento do setor agrícola face às inúmeras vantagens tecnológicas, pois há custos elevados de investimento em Tl que se não forem devidamente planejados, pouco repercutirão em incrementos de produtividade e de qualidade da matéria-prima produzida.

Assim, para que haja uma maior integração entre todos os elos da cadeia de comercialização é necessário que se estabeleça uma cooperação entre eles, ou seja, a filosofia de trabalho deve ser alterada para que, através da padronização de medidas, documentos, entre outros, sejam viabilizados tanto o comércio interno como a exportação de produtos ou serviços. Além disso, o entrosamento político é essencial para que medidas mais efetivas sejam tomadas, em termos de melhores condições de infra-estrutura, ambientais, de trânsito e de segurança, para garantir condições mínimas de atuação de todos os ramos de atividades comerciais ou afins.

\section{REFERÊNCIAS}

ALVES, D. S. Sistemas de informação geográfica. In: Geoprocessamento. São Paulo: Escola Politécnica/USP, 1990. p.66-78.

BARROS, G. S. C. Economia da comercialização agrícola. Piracicaba: FEALQ, 1987. 306p.

BLANCO, L. A. Como obter vantagens competitivas a partir da logística. Supermercado Moderno, v.27, n.1, p.81-86, 1996.

BOWERSOX, D. J.; CLOSS, D. J. Logistical management: the integrated supply chain process. 3.ed. New York: Mcgraw-Hill, 1996. $730 p$.

BRITO, E. P. Z. ECR - Efficiente consumer response: mudando a cultura empresarial. Logística Moderna, v.6, n.37, p.14-18, maio 1996.

CAIXETA FILHO, J. V. Transporte e logística no sistema agroindustrial. Preços Agrícolas, v.10, n.119, p.2-7, set. 1996.

CONFEDERAÇÃO NACIONAL DA INDÚSTRIA. Custo Brasil. Rio de Janeiro: CNI, 1995. 30p.

CUNHA OLIVEIRA, A. C. M. da Tecnologia 
de informação: competitividade e políticas públicas. Revista de Administração de Empresas, v.36, n.2, p.34-43, abr./jun. 1996.

DAVENPORT, T. H. Reengenharia de processos: como inovar na empresa através da tecnologia da informação. 4.ed. Rio de Janeiro: Campus, 1994. 391p.

ESTRADAS precárias oneram transporte. Logística Moderna, v.6, n.41, p.30-31, out. 1996.

FELICIANO NETO, A. Tecnologia da informação na logística. Revista Tecnologística, v.1, n.6, p.20-2, mar. 1996.

FERNANDES, A. A.; ALVES, M. M. Gerência estratégica da tecnologia da informação. Rio de Janeiro: LTC, 1992. 261p.

GONÇALVES, C. A.; GONÇALVES FILHO, C. Tecnologia da informação e marketing: como obter clientes e mercados. Revista de Administração de Empresas, v.35, n.4, p.2132, jul./ago. 1995.

GRAHAM, C. F.: NAZEM, S. M. From eletronic data interchange to efficient consumer response: new directions for the food insutry. Journal of Food Products marketing, v.3, n.2, p.39-47, 1996.

LAMBERT, D. M. Mais informação, menos estoque. Logística Moderna, v.5, n.32, p. 89, jul./ago, 1995.

LAURINDO, F. J. B. Estudo sobre o impacto da estruturação da tecnologia da informação na organização e administração das empresas. São Paulo, 1995. 157p. Dissertação (Mestrado) - Escola Politécnica, Universidade de São Paulo.

LONG, R. B. Impacts of competition on food production, processing and marketing. Journal of Food Products Marketing, v.3, n.3, p.83-100, 1996.

LOPES, I. Rastreadores aliam segurança a facilidades logísticas. Revista Tecnologística, v.2, n.13, p.30-40, out. 1996.

MAIS do que vender. Logística Moderna, v.6, n.39, p.24-28, ago. 1996.

MALIN, A. B. Economia e política de informação: novas visões da história. São Paulo em Perspectiva, v.8, n.4, p.9-18, out./dez. 1994.

McGAUGHEY JR., R. E.; SNYDER, C. A.; CARR, H. H. Implementing information technology for competitive advantage: risk management issues. Information \& Management, v.26, n.5, p.273-280, 1994.

MESQUITA, R. Informação sem limites. Problemas Brasileiros, v.2, n.310, p.25-29, jul./ ago. 1995.

MORGADO, E. M. Questões-chaves no gerenciamento da tecnologia de informação no setor bancário brasileiro. São Paulo, 1995. 110p. Tese (Doutorado) - Faculdade de Economia e Administração, Universidade de São Paulo.

O CLIENTE insatisfeito. Revista Tecnologística, v.2, n.11, p.54-56, ago. 1996.

OLIVEIRA, M. de. Até Scotland Yard vem ver a violência. Revista da Indústria, v.1, n.9, p.40-1, 02 set. 1996.

OXNER, W.; CHARLAB, S. A revolução da informação: artigos publicados no Jornal do Brasil. Rio de Janeiro: SENAI, 1995. 44p.

PIQUINI, M. O avanço da telemática. Transporte Moderno, v.28, n.321, p.20-23, nov. 1990. 
RAYMOND, L.; BERGERON, F. EDI sucess in small and medium-sized enterprises: a fiel study. Journal of Organizational Computing and Electronic Commerce, v.6, n.2, p.161-172, 1996.

SILVA, J. G. da. Impactos das tecnologias da informação na agricultura. Revista de Economia e Sociologia Rural, v.34, n.2, p.730, nov./dez. 1995.

SLATES, P. A. et al. Logistic chain modelling. European Journal of Operational Research, v.87, n.1, p.1-20, 16 nov. 1995.

STRASSMANN, P. A. Information payoff: the transformation of work in the eletronic age. New York: Free Press, 1985. 298p.

STREETER, D. H.; SONKA, S. T.; HUDSON, M. A. Information technology, coordination and competitiveness in the food and agribusiness sector. American Journal of Agricultural Economics, v.73, n.5, p.14641471, dec. 1991.

TECNOLOGIA acelera transporte rodoviário. O Estado de São Paulo, São Paulo, 24 set. 1995. p. B14.

TIGRE, P. B.; ROVERE, R. L. L.; FAGUNDES, J. Tecnologias da informação e desenvolvimento: novas evidências sobre sua difusão e impactos econômicos. Revista Brasileira de Economia, v.49, n.4, p.697-732, out./dez. 1995.

UM ANO de mudanças fundamentais. Logística Moderna, v.7, n.43, p.13, jan. 1997.

VANS traduzem o mundo do EDI. Revista Tecnologística, v.2, n.12, p.20-28, set. 1996.

VAREJISTAS em busca de eficiência. Logística Moderna, v.6, n.42, p.12-13, dez. 1996.
WALTON, R. E. Tecnologia de informação: o uso de TI pelas empresas que obtêm vantagem competitiva. São Paulo: Atlas, 1993. $215 p$.

WEITZEN, H. S. Poder da informação: como transformar a informação que você domina em um negócio lucrativo. São Paulo: Makron Books, 1991. 243p.

\section{Lilian Cristina Anefalos}

Engenheira Agrônoma, Pesquisadora Científica do Instituto de Economia Agrícola.

\section{José Vicente Caixeta Filho}

Engenheiro Civil, Professor-Associado do Departamento de Economia e Sociologia Rural da ESALQ/USP

\section{Title}

The Influence of Information Technology on the course of the commercialization of products

\section{Abstract}

This paper shows the current relevance of information technology, emphasizing some key sectors connected to the commercialization of products. The main developments and limitations of this technology combined with 
telecommunication resources are presented in the main links of some commercialization chains. The information technology has a great potential so it is important tohave the cooperation among the several sectors involved in this chain, in order to make use of this technology in all parts of commercialization.

\section{Keywords}

Satellite; Information Technology; Access to Information; Commercialization; Transportation.

\section{Titulo}

Tecnologia de información y su influencia sobre los rumbos de la comercialización de productos

\section{Resumen}

Muestra la importancia de la tecnología de la información en la actualidad, con énfasis en algunos sectores claves relativos a la comercialización de productos. Presenta los principales avances y limitaciones de la combinación de recursos de telecomunicaciones y de tecnología de información en los principales eslabones de algunas cadenas de comercialización. A pesar del gran potencial de esa nueva tecnología es importante que haya cooperación entre los diversos sectores de esa cadena para permitir su plena actuación en todos los ramos de la comercialización.

\section{Palabras-Clave}

Satélite; Tecnología de Información; Acceso a la Información; Centralización; Transporte

Artigo recebido em: 30/04/98 